\title{
DIELECTRIC-TO-DIELECTRIC CHARGING CHARACTERISTIC IN MEMS SWITCHES WITH CONTINUOUS AND DISCONTINUOUS CONTACT

\author{
D. Molinero $^{I^{*}}$, S. Cunningham ${ }^{l}$, D. DeReus ${ }^{l}$ and A. Morris ${ }^{2}$
} \\ ${ }^{1}$ Wispry, Inc, Irvine, California, USA
}

\begin{abstract}
A dielectric charging characterization is presented based on simple MEMS structures with continuous and discontinuous insulator-insulator contact. The amount of surface charging generated by triboelectric effects when both insulators are in contactis strongly dependent of the area of contact and electric field. Electrostatic simulation and hold-down measurements were done to characterize the surface charging on continuous and discontinuous teststructures that minimizes the contact area and electric field. The results have shown a considerable decrease of surface charge generation on discontinuous contact.
\end{abstract}

\section{INTRODUCTION}

The increase of mobile communication has driven the already busy and complex radio signal spectrum to a new level. The massive transfer of voice and data makes almost mandatory the use of tunable filters to maintain optimal signal strength values[1]. As the complexity and saturated spectrum increase, the demand of new tunable designs with extremely linear characteristic is needed. It is in this particular case where RF Micro-ElectroMechanical Systems (RF MEMS) can provide the necessary performance compared to conventional semiconductor devices. The high RF MEMS performance in linearity and quality factor are well-known [2]. Recently, integration of reliable RF MEMS with CMOS has propelled the devices into high volume mobile handsets [3]. The most significant characteristics of Wispry's RF MEMS-CMOS integrated tunable capacitor are its high linearity (IIP3 > 80dBm), high quality factor $(>100$ at $1 \mathrm{GHz})$, and long lifetime $(2.5 \mathrm{~B}$ cycles at $65{ }^{\circ} \mathrm{C}$ ), enabling use in a wide range of mobile handset applications [4]. Data communication increases over a wide range of bands in the radio spectrum are leading to increasing number of tuning events over the life of the handsets, which will require extended and improved tunable lifetime.

One of the main reliability issues of RF MEMS switches is dielectric charging [5]. The high electrostatic field needed to close the switch generates charge inside the dielectric layers causing undesired drift of the switch characteristic, hence deteriorating the tuning performance and leading, on the extreme case, to an irrecoverable stiction. The origin of dielectric charging varies depending on the location, type of contact and mechanism involved. Charging can occur in the dielectric bulk through injection from the electrodes into the dielectric with relatively quick charge/discharge dynamics. In the other hand, surface charging depends on the type of contact used. Metal-dielectric contact produces surface charge by injection when the switch is actuated while triboelectric effects will be the main mechanism when two dielectrics are in contact, where charge exchange can occur between dielectrics without injection from the metal due to both dielectric thicknesses [6]. In both cases, charging is a fast mechanism when the switch is actuated under high voltage, but discharge is comparatively slow when the voltage is removed. Once the surface charge is created and the contact is broken after removing the applied voltage, the only way for the charge to dissipate is through diffusion across the thickness of the dielectric, which is difficult because bulk diffusivity is low in high-quality dielectric. Therefore, surface charging is particularly detrimental because, once the charge is generated on the surface, it cannot be removed, and cumulative deterioration will take place.

Previous work has shown how bulk charging is minimal in our test structures; however, surface charging by triboelectric effects has an important role, being the key factor limiting the switch lifetime [6]. Although triboelectric effects have been studied for centuries, and while the physical mechanism involved on the charge exchange is not always, it is understood that ion or electron transfer at contact leads to charge generation in MEMS switches. Many parameters can contribute to surface dielectric charging, such as the dielectric materials used, the fabrication process and the ambient operating conditions [7], which can be difficult to control. Whether electrons or ions are present in the charge exchange, reducing the electric field and area of contact will mitigate the amount of charge accumulation. It is well-known that the triboelectric effect is strongly proportional to the area of contact, and the energy associated to transfer ions or electrons to the electric field [7]. Therefore, by reducing both parameters will assure a reduction of the rate of charging and the total amount of charge generated, with the consequential lifetime increase.

In this paper, we show how to reduce the amount of charging by the inclusion of spatially distributed bumpers associated with a patterned metal that reduces the electric field in the area of contact. Representative simulations and measurements are provided for comparison over a range of voltages and temperatures for various designs.

\section{SIMULATIONS}

The inclusion of insulating bumpers reduces the contact area and minimizes the surface charge generation. However, the benefit of such bumpers alone may not be sufficient for newer reliability requirements, particularly at high temperature. In this particular analysis, electrostatic simulations were performed to study the impact of a patterned top metal hole over the bumper area to reduce the electrostatic field.

Simulations were done with Matlab (C) PDE toolbox [8], where a single bumper was characterized. The lateral dimensions were chosen as the same as the bumper pitch to enable use of periodic boundary conditions. Fig. 1(a) shows the simulated crosssection used to simulate the electrostatic field. A permanent gap of $4 \mathrm{~nm}$ was intentionally left between the bumper zone and the lower dielectric to emulate the effect of surface roughness when in contact. It is theorized that the electric field in this residual gap is the key driver for the charge transfer. Fig. 1(b) and 1(c) show the solution on a particular case of a $1400 \mathrm{~nm}$ patterned metal hole. It can be seen that the maximum electric field both under the bumper and in the parallel plate area away from the bumper have values around $3 \mathrm{MV} / \mathrm{cm}$. However, surface charge is only generated under the bumper where intimate contact occurs.

Fig. 2(a) shows the surface electrostatic field near the bumper for different hole dimensions, where the inclusion of a bumper with no metal pattern reduces the electrostatic field from 10.5 to 8 


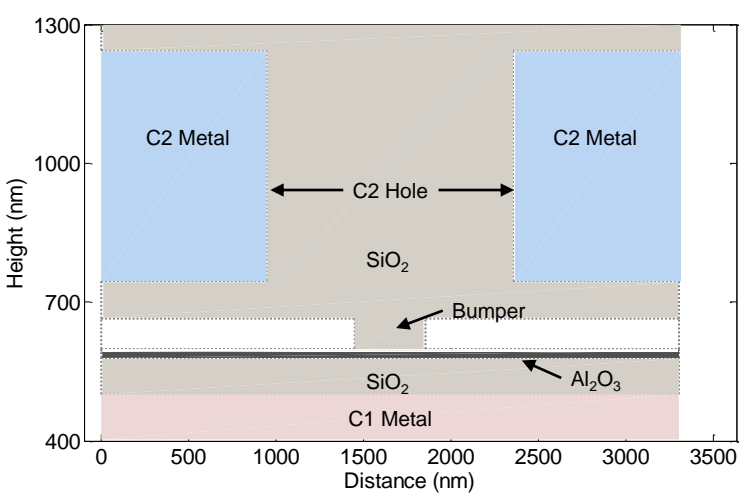

(a)

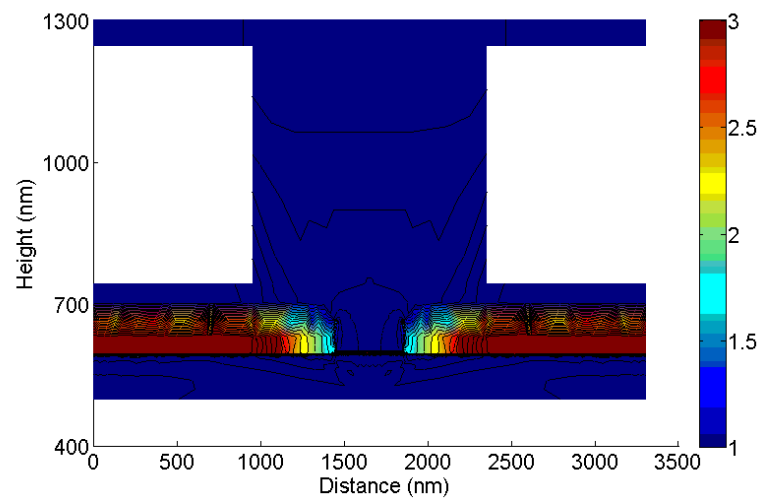

(b)

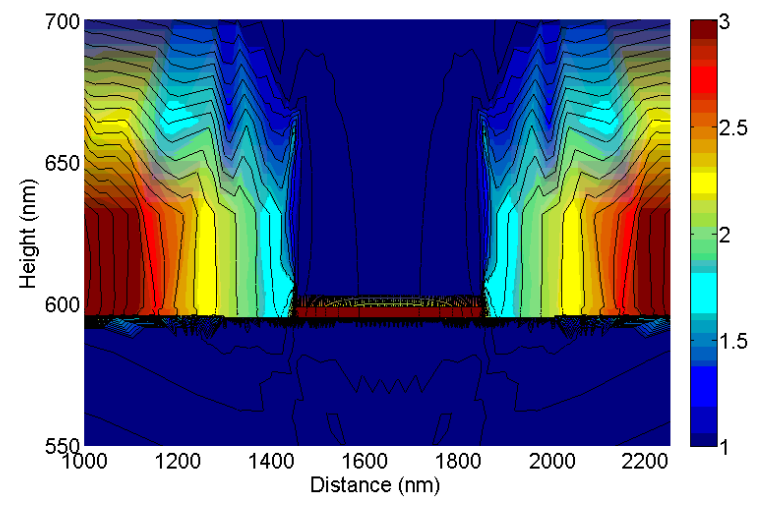

(c)

Figure 1: (a) Cross-section of the simulated test structure defined in the Matlab ${ }^{(}$PDE Toolbox and (b) the electrostatic result for a given bumper/C2 Hole parameter. (c) Close up view of the electrostatic field surrounding the bumper zone.

$\mathrm{MV} / \mathrm{cm}$. As the metal hole diameter increases, the electrostatic field is reduced. Fig. 2(b) shows the electrostatic variation as function of the metal hole diameter under different voltages. Simulations have shown two clear sections depending on the metal hole dimensions: a fast transition from high to low field when the hole and the bumper dimensions are comparable, and a semi steady state condition when the lateral dimensions of the hole are larger than the bump. Based on these simulation results, the optimal metal pattern diameter should be at least $3 \mathrm{x}$ times larger than the bumper dimension. Even larger dimensions will not provide significant change when balanced against the increase in actuation voltages when the hole area becomes comparable to the initial gap

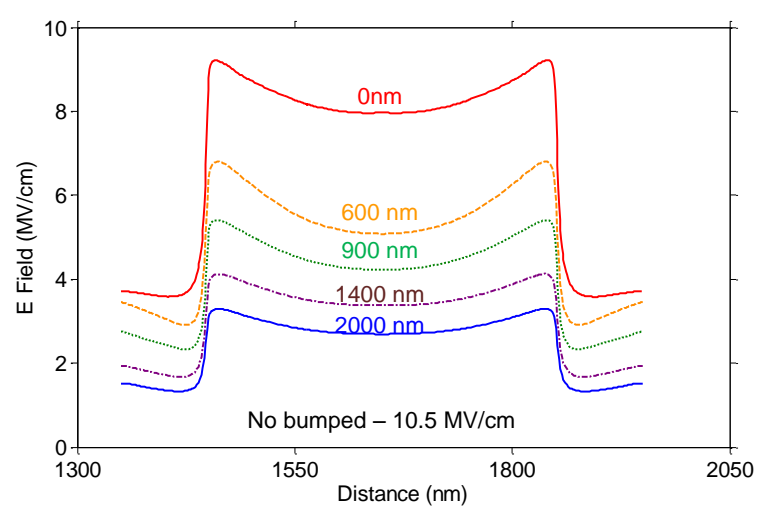

(a)

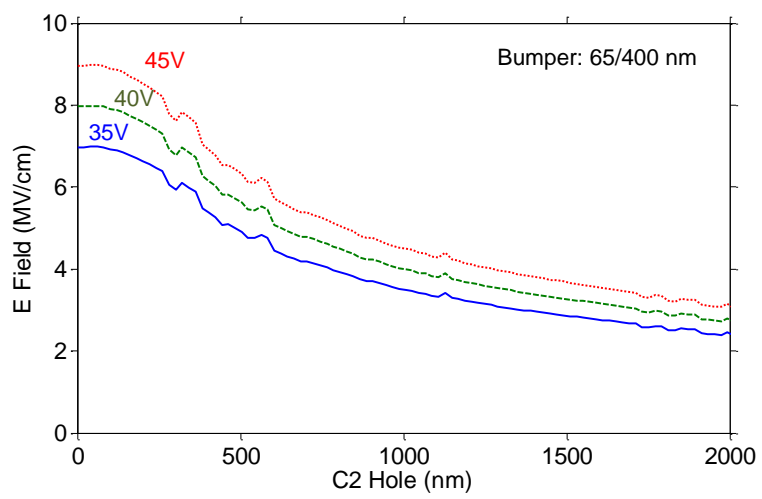

(b)

Figure 2: (a) Surface Electrostatic field under the bumper are for different top metal hole diameter, and (b) its center value as function of different top metal hole diameter and voltage.

$(2 \mu \mathrm{m})$ [9]. For the experimental study, a $1400 \mathrm{~nm}$ metal diameter was chosen.

\section{FABRICATION AND EXPERIMENTAL}

Two test structures were designed to intentionally monitor dielectric charging while being fabricated alongside MEMS capacitive switch array products on the same hermetically sealed wafer using a $0.18-\mu \mathrm{m}, 5 \mathrm{~V}$ CMOS/50 V LDMOS process [10]. Electrostatic cantilever-type MEMS capacitors were used for the test structures, which were formed with the final three $\mathrm{AlCu}$ metal levels in chemical vapor deposited $\mathrm{SiO}_{2}$ dielectric to form a metaloxide-metal composite structure. The MEMS cavities were formed using a planar sacrificial silicon process that is processed at less than $400^{\circ} \mathrm{C}$. The cantilever consists of $\mathrm{a} \mathrm{SiO}_{2}$ sandwiched by thin $\mathrm{AlCu}$ above and below. The capacitor electrodes are formed between the lower $\mathrm{AlCu}$ of the cantilever and the $\mathrm{AlCu}$ at the bottom of the lower cavity, where the lower metal is covered by thin layers of $\mathrm{SiO}_{2}$ and $\mathrm{Al}_{2} \mathrm{O}_{3}$ and the upper metal is covered only by $\mathrm{SiO}_{2}$ (Fig. 3(a)). Bumpers are made of the same $\mathrm{SiO}_{2}$ that is under the dielectric top electrode. In this particular case, bumpers were $65 \mathrm{~nm}$ tall, $400 \mathrm{~nm}$ wide and spaced by $3300 \mathrm{~nm}$ from each other.

Both test structures have active area of approximately 40x40 $\mu \mathrm{m}^{2}$ with similar pull-in and release voltages of approximately $20 \mathrm{~V}$ and $10 \mathrm{~V}$, respectively. The capacitance ratio is drastically reduced on the discontinuous test structure due to the bumpers. Fig. 3(b) shows the capacitance - voltage $(\mathrm{C}-\mathrm{V})$ of both structures. 


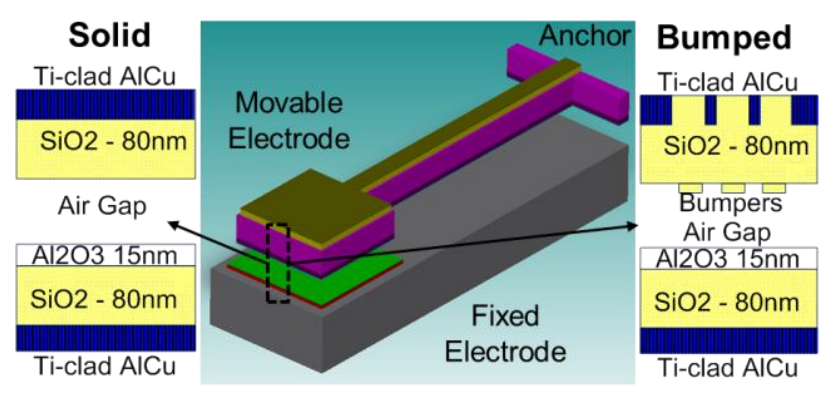

(a)

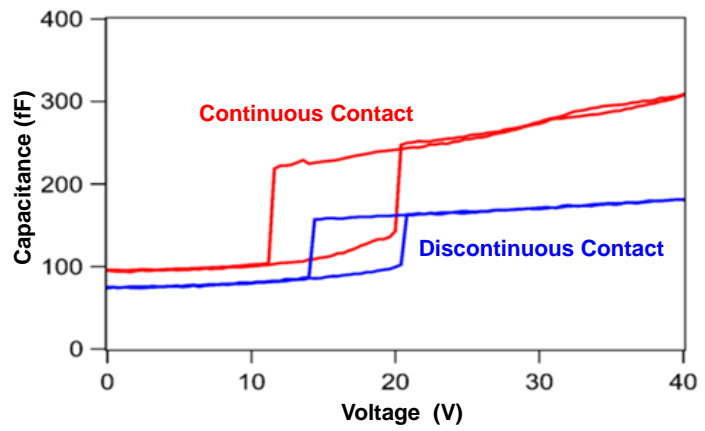

(b)

Figure 3: (a) Schematic cross-section of Wispry's dielectric charging continuous and discontinuous test structure. The cantilever consists of $\mathrm{SiO}_{2}$ sandwiched by thin $\mathrm{AlCu}$ above and below. The capacitor electrodes are formed between the lower $\mathrm{AlCu}$ of the cantilever and the $\mathrm{AlCu}$ at the bottom of the lower cavity, both of which are coated with thin layers of $\mathrm{SiO}_{2}$ and $\mathrm{Al}_{2} \mathrm{O}_{3}$. (b) Capacitance-Voltage $(C-V)$ sweeps of both test structures.

The characterization was performed on wafer with an Agilent E4980A LCR meter that was used to monitor the capacitance values at the specific voltage provided by the Keithley 2400 source. An appropriate bias tee was used to isolate the DC signal from the LCR meter. The measured capacitance was calibrated at the probe tips. The setup and measurement extraction were controlled by an external PC and Labview program. The charging characterization consisted of applying a constant DC hold-down voltage for $100 \mathrm{~min}$. A triangular sweep was used on logarithmic intervals during the hold-down stress to measure the $\mathrm{C}-\mathrm{V}$ characteristics and extract the pull-in and release voltages.

\section{RESULTS}

Fig. 4 shows the measurements results of both test structures topologies under different hold-down voltages and temperature. The actuation voltage shifts in both devices increase at any holddown voltage and remains unaltered once the applied voltage is removed, providing a clear indication of surface charging and minimal or non-existent bulk charging. The transient characteristic shows a clear impact of the discontinuous contact versus the continuous contact test structure, where the amount of surface charge generated has decreased notably. Both test structures show a different transient performance, while the continuous contact has a fast early build up followed by a more gentle charging behavior, the discontinuous contact shows a more linear increase of the surface charge. The actuation voltage shift transients also present a different behavior at elevated temperatures. The continuous contact presents less variation of the actuation voltage shift as the temperature increase, but a faster transition. On the other hand, the discontinuous contact shows a slight increase in both the final state and transition. This behavior can be attributed to two situations:

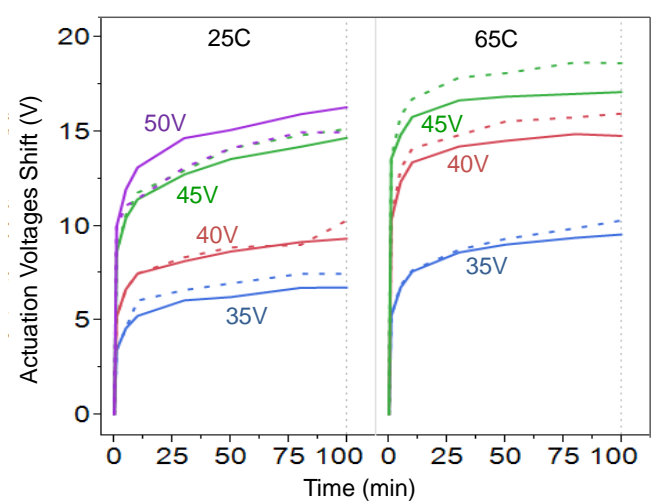

(a)

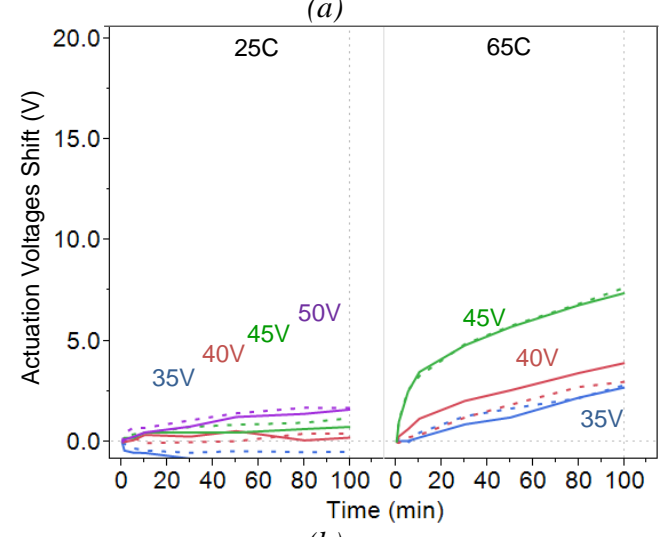

(b)

Figure 4: Pull-in an (solid) and release (dash) voltage shift for continuous (a) and discontinuous (b) contact at different voltages and temperatures. Bumpers and top metal holes reduced the surface charging considerably and hence improved switch lifetime.

first, the reduced electric field and contact delays the charging characteristic seen in the continuous devices, and second, the resulting charge distribution will modify in a different manner the actuation voltage shift [911].

The final actuation voltage shift presents a better evaluation of the improvement due to the bumped contact and patterned top metal. Fig. 5 shows the actuation voltage shift results at $100 \mathrm{~min}$ under a wide range hold-down voltages and temperature. The results show a clear reduction of the amount of surface charging between the two test structures. A linear characteristic is extracted with different slopes over voltage, enhanced by elevated temperature. It should be pointed out that the results of the continuous contact are similar in any given point of the hold-down stress but with less voltage shift, indicating a unique mechanism behind the surface charge generation. In the discontinuous contact, the same approach can be taken but for longer times. At short times, the slope at room temperature is difficult to acquire, however, at elevated temperature shows a decrease of the voltage dependence. The measured data is not enough to indicate that two different mechanisms are involved on the surface charge generation, and we attribute this effect to the charge distribution being created from the bumper inclusion. This explanation can also be attributed to the differences in the linear response over voltage of the actuation voltages shift. The charge generation mechanism in both devices are the same and attributed to triboelectric effects, moreover, both test structure were fabricated on the same wafer, and hence having similar fabrication process and impurities associated with it. The charge generation mechanisms are the same but have reduced impact in the discontinuous contact due to the 


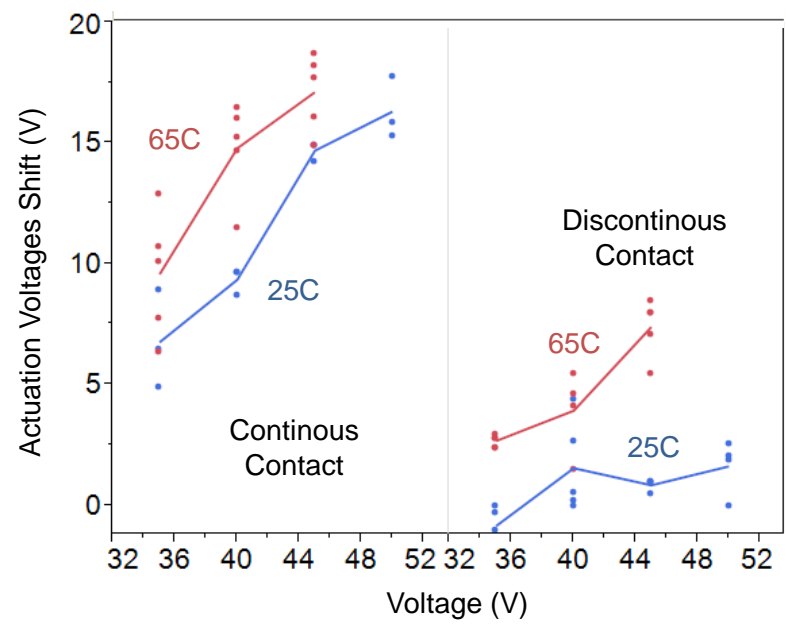

Figure 5: Actuation voltage shift after 100 min hold down test for continuous and discontinuous contact. Both results show a linear characteristic with significantly lower value and slope for the discontinuous contact.

inclusion of bumpers and the reduced electrostatic field. Note that the resulting charge generation also has a lateral distribution corresponding to the bumper pattern, which leads to non-uniform charge and charge evolution across the surface which affects the actuation voltage shift characteristic [911].

Based on the test structure design and considering a homogenous surface charge, the maximum charge density can be calculated as follow

$$
q\left(\mathrm{C} / \mathrm{m}^{2}\right)=\Delta V_{P, R} \cdot \epsilon_{0} /\left(\frac{t_{S}}{\epsilon_{S}}+\frac{t_{A}}{\epsilon_{A}}\right)
$$

Where $\varepsilon_{0}$ is the air relative permittivity, $\varepsilon_{\mathrm{S}}$ and $\varepsilon_{\mathrm{A}}$ are the silicon oxide and alumina relative permittivity, $t_{\mathrm{S}}$ and $t_{\mathrm{A}}$ are the dielectric thickness. Considering a normal operation at $40 \mathrm{~V}$ at elevated temperature, the total amount of charge density extracted after the $100 \mathrm{~min}$ hold down test for the continuous and discontinuous contact is about 6 and $1.6 \mathrm{mC} / \mathrm{m}^{2}$ respectively. While the discontinuous contact and patterned metal reduce the charge significantly, work continues to reduce even more the amount of charge for future applications.

\section{CONCLUSION}

In conclusion, a full study based on simulations and experimental data is shown for capacitive MEMS test structures with continuous and discontinuous contact. It has been shown how the inclusion of bumpers considerably reduces the amount of charge created by triboelectric effects. However, bumpers alone are not sufficient to achieve certain reliability criteria. For this purpose, electrostatic simulations were performed to study the effects of a metal pattern on one of the electrodes to reduce the electrostatic field in the contact area. The results showed that the inclusion of a bumper only reduces the electrostatic field from 10 to $8 \mathrm{MV} / \mathrm{cm}$, but adding a top metal pattern reduces this much further to around $4 \mathrm{MV} / \mathrm{cm}$. The results have shown a top metal pattern of about $3 \mathrm{x}$ larger than the bumper size is an effective compromise between charging reduction and actuation area. In this experiment, a $1400 \mathrm{~nm}$ metal pattern was chosen, with minimal change on the actuation voltage as the value is smaller than the initial gap.

Experimental data comparing both test structures with continuous and discontinuous contacts were performed. The results show that the discontinuous contact decreases notably the amount of charge generated. A change in the transient characteristic is clearly appreciated, but mostly due to the reduction of the amount of charge and its distribution over the surface due to the bumpers contact. Both test structures follow a linear relationship with voltage; however, the slope is different due to the electrostatic field reduction from the top metal pattern. Overall, adding the metal pattern significantly improves the reliability capability of RF MEMS capacitors in terms of dielectric charging.

\section{REFERENCES}

[1] J.L. Hilbert, "RF-MEMS for Wireless Communications," Communications Magazine, IEEE, vol.46, no.8, pp.68-74, August 2008.

[2] G. M. Rebeiz, RF MEMS Theory Design Technology. Hoboken: Wiley, 2003.

[3] A. S. Morris, S. P. Natarajan, Q. Gu, and V. Steel, "Impedance tuners for handsets utilizing high-volume RFMEMS," in Proc. European Microwave Conf., Oct.-Nov. 2012, pp. 193-196.

[4] www.wispry.com

[5] W. M. van Spengen, "Capacitive RF MEMS switch dielectric charging and reliability: a critical review with recommendations". J. Micromechanics \& Microengineering, vol. 22, no. 7, pp. 1-23, June 2012.

[6] D. Molinero et al.,'Dielectric charging in MEMS with dielectric-dielectric contacts", Transducers \& Eurosensors XXVII: The 17th Int. Conf. on Solid State Sensors, Actuators and Microsystems, pp.1400 -1403, 16-20 June 2013.

[7] D. J. Lacks and R. M. Sankaran, "Contact electrification of insulating materials", J. Phys. D: Appl. Phys. 44, 453001 (2011).

[8] http://www.mathworks.com/products/pde/

[9] G. M. Rebeiz, RF MEMS, Theory, Design. And Technology, John Wiley and Sons Publications, 2003.

[10] A.K. Stamper et al., "Planar MEMS RF capacitor integration" Transducers 2011, 16th International,pp.1803-1806, 5-9 June 2011.

[11] X. Rottenberg et al.,'Distributed dielectric charging and its impact on RF MEMS devices", in Proc. European Microwave Conf., Oct., 2004, pp. 77-80.

\section{CONTACT}

*D. Molinero, tel: +1-949-334-8825; david.molinero@wispry.com 